\title{
Tree Approximation for Spin Glass Models
}

\author{
Naoki Kawashima \\ Institute for Solid State Physics, University of Tokyo, Kashiwa, Chiba 277-8581, Japan
}

\begin{abstract}
An approximate numerical approach to spin models is proposed, in which the original lattice is transformed into a tree. This method is applied to the Edwards-Anderson spin glass model in two and three dimensions. It captures the characteristics of each individual sample and reproduces various qualitative features of sample averaged quantities similar to those that have been observed in previous Monte Carlo simulations. For example, from the Binder parameter for various system sizes as a function of the temperature we obtain $T \sim 1.0$ with $\nu \sim 1.85$, in reasonable agreement with previous Monte Carlo simulations. The present approximation yields the trivial structure for the overlap distribution function.
\end{abstract}

KEYWORDS: spin glass, real-space renormalization, Migdal-Kadanoff approximation, Cayley tree

\section{Introduction}

The spin glass problem was brought forth by experiments on random magnets ${ }^{1}$ and the mathematical model was subsequently proposed. ${ }^{2}$ Since then, the simplicity of the model and the fundamental nature of the problem have been encouraging the investment of a great deal of effort on its study. While the existence of the spin-glass phase transition was established by Monte Carlo simulations $^{3-5}$ for the three-dimensional case, the absense of a phase transition in two dimensions was more difficult to conclude and it was settled ${ }^{6,7}$ after the threedimensional problem was solved. Concerning the nature of the spin glass phase in three dimensions, early theoretical works produced two paradigms that are consistent with experimental findings: the mean-field picture ${ }^{8}$ and the droplet picture. ${ }^{9}$ Therefore, investigations in the last two decades have been focused on the question as to which paradigm is correct for spin glass models in two and three dimensions. For solving this problem, the principal tool was numerical calculations. It did not take too long, however, for researchers to realize the difficulty of the problem from the computational point of view. Even a mathematically rigorous statement was made: ${ }^{10}$ the problem of finding the ground state of a given sample of the Edwards-Anderson spin glass model is NP-hard if the lattice is three- or higher-dimensional.

While this mathematical fact does not necessarily exclude the possibility of finite-temperature calculation within a reasonable amount of computational time, no numerical method has proved to be polynomial-time bounded, in particular, for temperatures below the critical point. To this day, the largest size of the threedimensional system of which the equilibrium properties can be studied within a realistic computational time does not exceed $L=30$, which is not enough to settle the various important issues beyond reasonable doubt. The situation is rather discouraging because it is numerically hopeless to push much further along the same line of effort if the difficulty is a direct consequence of the NPhardness of the ground state problem, as facts seem to point.

In the present article, therefore, we would like to call
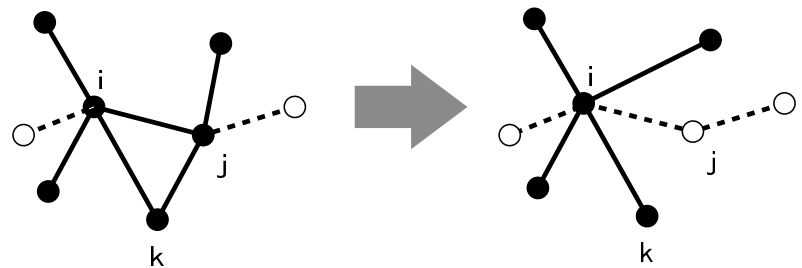

Fig. 1. Contraction process. Circles are vertices and lines are edges. Solid lines are those which are still in the list and dashed lines are the ones that have been 'contracted' and eliminated from the list.

a renewed attention to another method for approaching the issue, i.e., an approximate numerical method. One of the most well-known examples for methods of this kind is the Migdal-Kadanoff (MK) real-space renormalization, ${ }^{11}$ which yields a reasonably accurate result for the ferromagnetic Ising model and was applied later to spin glasses. ${ }^{12,13}$ The procedure is often associated with the domain-wall renormalization group ${ }^{14-16}$ in which the domain-wall excitation free energy is identified with the effective coupling in the renormalized scale. It is, then, related to the droplet picture ${ }^{9}$ by associating the domainwall excitation to a droplet of the same scale.

The procedure of the MK renormalization consists of two steps: bundling bonds and tracing spins out. The approximation is intuitively justified for the ferromagnetic system up to the correlation length because in a region of a size smaller than the correlation length most spins are parallel to each other and therefore the effect of $n$ parallel couplings in that region would be the same as a single stronger coupling that connects two neighboring regions. Since this is not even qualitatively true for the spin glass systems due to their high inhomogeneity, the validity of the approximation is somewhat more questionable there. Nonetheless, the approximation seems to work well for spin glasses ${ }^{12,13}$ judging from the fact that it produces results consistent with other numerical findings, such as the (likely) absence of phase transition in two dimensions, ${ }^{14,15}$ and its existence in three dimensions. ${ }^{5}$

A disadvantage of the MK procedure, however, is that 

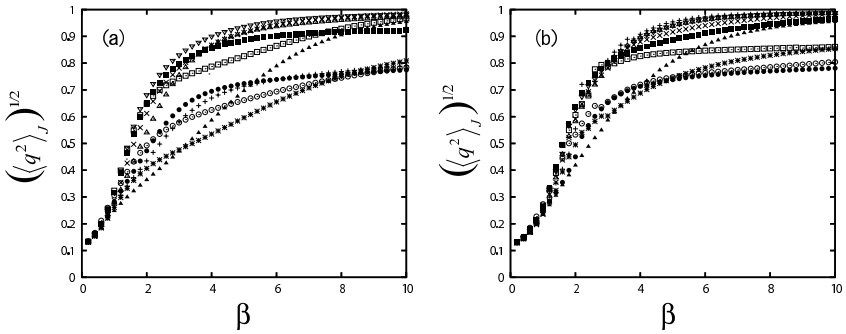

Fig. 2. The standard deviation of the overlap distribution function, $P_{J}(q)$, for 10 randomly generated samples of an $8 \times 8$ system. The exact values are on the left panel, (a), and the result of the present approximation is on the right, (b). The same bond samples are shown with the same symbols in both the panels.
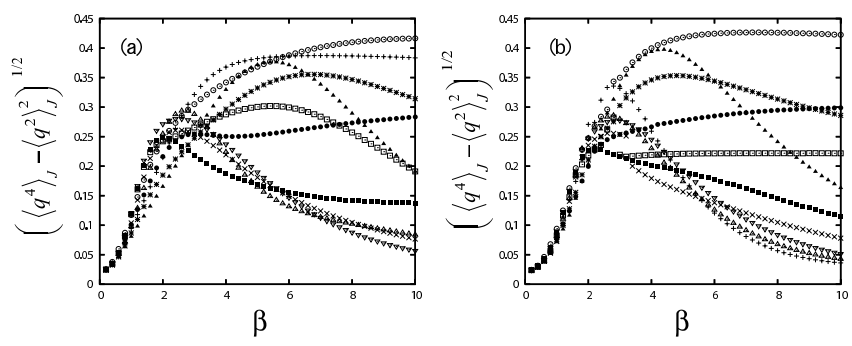

Fig. 3. The standard deviation of the squared overlaps. The same samples as Fig. 1.

one cannot obtain results for a finite system with a given bond configuration, say, in three dimensions. This is due to the lack of direct correspondence between the original spins in finite dimensions and the spins in the MK procedure. This is one of the motivations that drive us to investigate a new procedure while the main motivation is to improve the quality of the approximation taking into account the inhomogeneity of the system.

\section{Procedure}

We start from the graph of the same structure as the finite lattice on which the model is defined. The vertices of the graph correspond to spins and edges to bonds. We gradually deform the graph by contraction processes discussed below until it becomes a tree with no loop. Before starting the contraction processes, a vertex initially carries the volume of unity and an edge carries the weight equal to the coupling constant of the corresponding bond. The volumes of vertices and the weights of edges may be changed during a contraction process. All the edges are initially enlisted as the candidates for contraction.

The graph deformation is accomplished by applying the following contraction procedure repeatedly to the heaviest edge in the list until the list becomes empty. Here, the heaviest edge is the one with the weight of the largest absolute value. Let $e$ be the edge and $i$ and $j$ the two vertices connected by $e$, where $i$ has a larger volume than $j$. Then, we let $i$ enslave $j$. This is done by detaching all the edges connecting $j$ to other vertices, except $e=(i j)$, and reconnect them to $i$. (Fig. 1) For a vertex $k$ that is connected to $j$ before the reconnection, the new weight of the edge $(i k)$ after reconnection

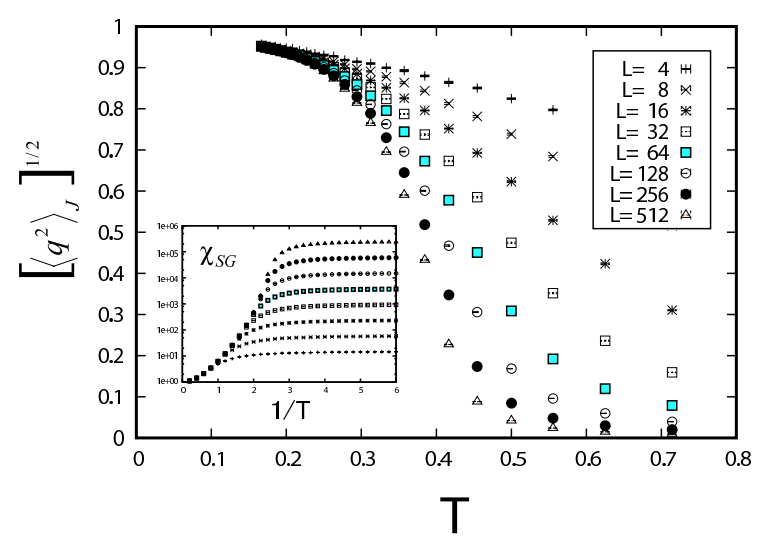

Fig. 4. The mean overlap $\sqrt{\left[\left\langle q^{2}\right\rangle_{J}\right]}$ in two dimensions. The inset is the spin glass susceptibility $\chi_{\mathrm{sg}} \equiv L^{d}\left[\left\langle q^{2}\right\rangle_{J}\right]$ in the logarithmic scale. ( $L=4$ and 8 are not shown in the main panel.)

is $J_{i k}^{\text {new }} \equiv J_{i k}^{\text {old }}+J_{i k}^{\prime}$, where $J_{i k}^{\prime}$ is defined by

$$
\tanh \left(\beta J_{i k}^{\prime}\right) \equiv \tanh \left(\beta J_{i j}\right) \times \tanh \left(\beta J_{j k}\right)
$$

with $\beta$ being the inverse temperature. (If $i$ and $k$ are not directly connected before the reconnection, $J_{i k}^{\text {old }}$ is simply zero.) The volume of the vertex $i$ is updated simply as $V_{i}^{\text {new }} \equiv V_{i}^{\text {old }}+V_{j}^{\text {old }}$. Then, the edge $e$ is removed from the list, which is the end of the contraction procedure of the edge $e$.

By every contraction process, the number of edges is reduced at least by one, and by definition of the procedure the contracted part (consisting of dashed lines and open circles in Fig. 1) does not have loops. Therefore, when all the edges in the list have been contracted, we are left with a tree. Once we obtain the tree, the spin-spin correlation function can be computed as the product of "nearest-neighbor" correlation functions along the path leading from one spin to the other:

$$
\Gamma_{i j} \equiv\left\langle S_{i} S_{j}\right\rangle=\prod_{e \in \pi_{i \rightarrow j}} \tanh \left(\beta \tilde{J}_{e}\right),
$$

where $\pi_{i \rightarrow j}$ is the path on the tree from $i$ to $j$, and $\tilde{J}_{e}$ is the weight of the edge $e$ on the tree. From correlation functions, we can obtain various quantities of interest, such as the spin glass susceptibility and the Binder parameter, as we present in what follows. ${ }^{17}$

Here, a few remarks on the validity of the approximation may be due. We should first note that the procedure yields exact results in one dimension. In two or higher dimensions, we must treat the results of the approximation with greater caution. Generally, we expect the present procedure to produce more accurate results when the distribution function of the couplings is broader. An approximation similar to the present one was proposed for quantum systems. ${ }^{18}$ It was shown to be asymptotically exact for a few one-dimensional systems ${ }^{19}$ and applied also to two-dimensional systems. ${ }^{20,21}$ This was possible because the width of the distribution diverges as we proceed in the renormalization transformation. Since the same is not true in the present case, the judgment of the validity or the utility of the present approximation must be based on what we actually obtain from it. We 


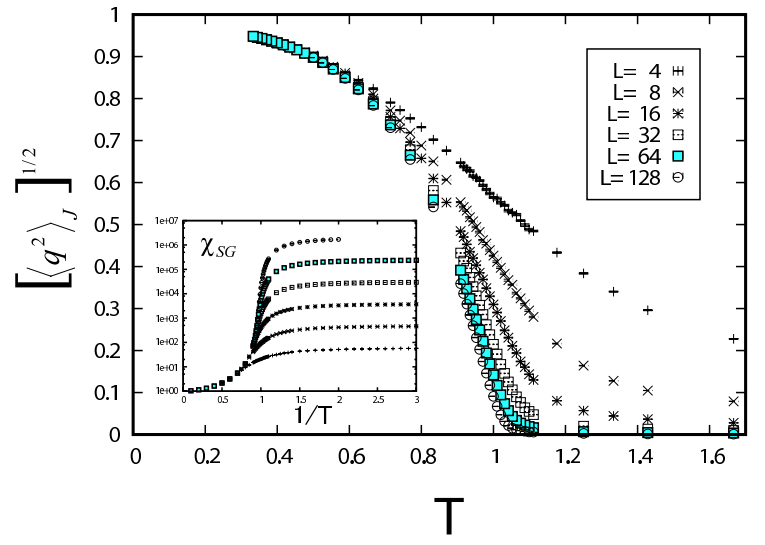

Fig. 5. The same as Fig. 4 for the three-dimensional case.

may, however, say that the present scheme is expected to be more accurate in lower dimensions when compared at the same temperature, because in order for the present approximation to be accurate the largest bond must be dominating among those which share the same spin, the situation occurring less likely for a higher coordination number.

\section{Individual Samples}

In order to obtain a rough idea as to what degree the approximation is accurate for each individual bond sample, we first compute the variances of the overlap $q$ and the squared overlap $q^{2}$, where $q$ is defined as $q \equiv \frac{1}{N} \sum_{i=1}^{N} S_{i}^{(1)} S_{i}^{(2)}$ with two replicas with the same bond configuration but independent spins. (The symbol $S_{i}^{(\alpha)}$ denote a spin at the site $i$ of the $\alpha$-th replica.) Of crutial importance is the distribution of $q$,

$$
P_{J}(q) \equiv\left\langle\delta\left(q-\frac{1}{N} \sum_{i=1}^{N} S_{i}^{(1)} S_{i}^{(2)}\right)\right\rangle_{J},
$$

with $J$ specifying a particular bond configuration, and its average over $J$, i.e., $P(q) \equiv\left[P_{J}(q)\right]$. The square brackets $[\cdots]$ denote the average over bond configurations hereafter.

The distribution $P_{J}(q)$ having a nontrivial structure is regarded as a defining character of the mean-field picture whereas in the droplet picture $P_{J}(q)$ is supposed to be trivial, i.e., a superposition of two delta functions at symmetric positions. In both pictures, the spin glass phase is characterized by the finite variance of $P_{J}(q)$. Therefore, the phase transition from the high-temperature paramagnetic phase to the low-temperature spin glass phase, can be detected in the behavior of the variance of $P_{J}(q)$. In Fig. 2, the standard deviation is shown for ten randomly chosen bond samples of two-dimensional EA spin glass model. The bond distribution is a Gaussian with a mean value of zero and a variance of unity.

The nature of the low-temperature phase may be better reflected in the behavior of the variance of the distribution of the squared overlap $q^{2}$, because the finite variance $\left\langle q^{4}\right\rangle_{J}-\left\langle q^{2}\right\rangle_{J}^{2}$ would be the most direct measure of the nontriviality of $P_{J}(q)$. The estimates of the quantity are shown in Fig. 3 for the same 10 bond samples as

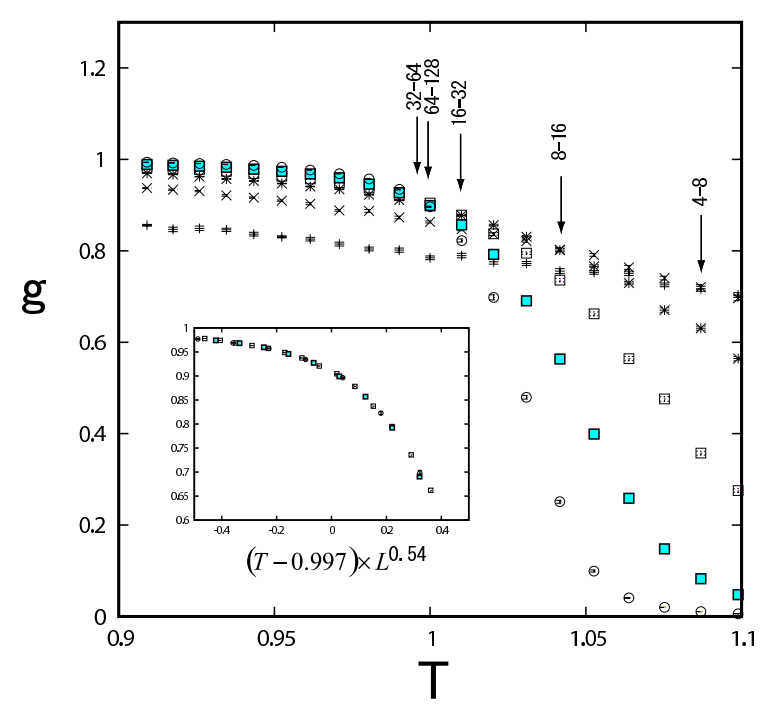

Fig. 6. The Binder parameter for the three-dimensional model of system sizes of $L=4,8,16,32,64$ and 128 (from top to bottom at high temperature). The arrows indicate the locations of the crossings between two successive system sizes. The inset shows the finite size scaling for the largest three sizes, $L=32,64$ and 128. (The other sizes are not shown in the inset.)

in Fig. 2.

Looking at the two sets of figures, we notice that the agreement between the left panels (exact calculation) and the right panels (approximation) is markedly good for a crude approximation like the present one. The locations of peaks and inflexion points in the right panel (present approximation) roughly agree with those in the left panel (exact) for most samples, suggesting that the approximation captures the characteristic features in the temperature profile.

\section{Sample Averages}

We now turn to the behaviors of averaged quantities. In Fig. 4, we plot the standard deviation of $P_{J}(q)$ averaged over bond configurations for the two-dimensional system. The inset is the spin glass susceptibility $\chi_{\mathrm{sg}} \equiv$ $N\left[\left\langle q^{2}\right\rangle_{J}\right]$ against the inverse temperature $\beta$. The envelope function of $\chi_{\mathrm{sg}}$ in the region $\beta<1$ shows roughly the same behavior as the previous computation, ${ }^{7}$ which is free from any approximation. Whereas the zero temperature phase transition was concluded in the previous exact computation, it is difficult to judge from the present figure whether the transition temperature is finite or not. The envelope function could be fit by a function for which the most singular term is $\exp \left(a \beta^{2}\right)$. Equally plausible is a function that diverges around $\beta \sim 3.5$. The previous exact computation shows that the susceptibility obeys the power law $\chi_{\mathrm{sg}} \propto T^{-2 \nu}$, where $\nu \sim 2.5,{ }^{7}$ in the temperature range $1.5<\beta<2.5$. In the same temperature range, the present envelope function (in the double logarithmic scale) is steeper. Therefore, we may not trust the critical exponent estimated from the slope of the present envelope function.

For the three-dimensional system, the spin glass susceptibility shows a larger change (Fig. 5) near $T \sim 1$, which is presumably the transition point. In this case, 


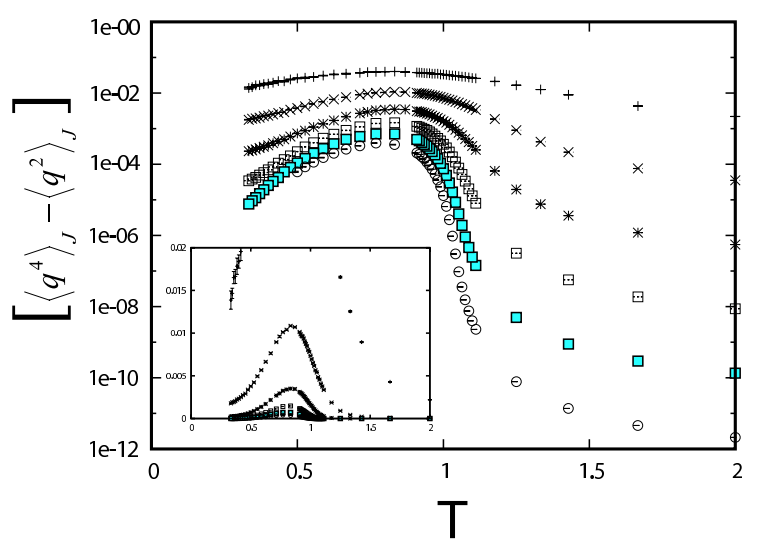

Fig. 7. The variance of the distribution of $q^{2}$ for the threedimensional model of $L=4,8,16,32,64$ and 128 (from top to bottom) in the logarithmic scale. The same quantity is plotted in the linear scale in the inset.

we would have to assume an even more singular function than $\exp \left(a \beta^{2}\right)$ in order not to allow a divergence at a finite temperature. It seems much more natural to assume a finite temperature transition, as is consistent with Monte Carlo simulations. ${ }^{3-5}$ In Fig. 6, the Binder parameter, $g \equiv\left[\left(3-\left\langle q^{4}\right\rangle_{J} /\left\langle q^{2}\right\rangle_{J}^{2}\right)\right] / 2$, is plotted against the temperature. Curves of various system sizes cross each other and the crossing between larger systems takes place at lower temperature, as is the case with the twodimensional model (not shown). However, the location seems to converge to some finite value in the thermodynamic limit, in contrast the two-dimensional case. In particular, the value of $g$ at the crossings increases in $g$ up to the $L \sim 32$, while we do not observe a statistically significant increase above this scale. The crossing point in the thermodynamic limit is located around $T \sim 1.0$ and $g \sim 0.9$. We can also produce a finite-sizescaling plot that looks reasonable, as shown in the inset of Fig. 6. The plot yields the estimates of the critical exponent; $\nu \sim 1.85$. The agreement of these values with the results of Monte Carlo simulation, $T_{c}=0.98(5)$ and $\nu=2.00(15),{ }^{22}$ are markedly good considering that the crudeness of the present approximation. This is also an improvement on the MK result, ${ }^{13} T_{\mathrm{c}} \sim 0.39$ and $\nu \sim 2.8$.

In Fig. 7, the variance of $q^{2}$, averaged over bond configurations, is shown in a logarithmic scale. (The same is presented in a linear scale in the inset.) The variance is a decreasing function of the system size at any temperature. The decrease is rapid both at the high and low temperature ranges and is somewhat more moderate in the middle. However, even at the temperature where the system size dependence is the weakest, the variance is extremely small and seems to converge to zero in the thermodynamic limit, consistent with the droplet scenario.

\section{Concluding Remarks}

We have proposed a simple procedure that is motivated by the real-space renormalization-group methods of MK and analogous to that of Dasgupta, Ma and Hu. ${ }^{18}$ It produces a self-consistent framework for understand- ing spin glass systems and makes it possible to compute various quantities, including $P(q)$, for a large given sample in finite dimensions, which is otherwise impossible to compute. The results for three-dimensional systems show an unexpectedly good agreement with the other numerical methods that do not involve any approximation, while the low-temperature behaviour in two dimensions shows the limitation of the present approximation. As for the triviality/nontriviality of $P(q)$ in the spin glass phase, the approximation supports the trivial structure. This is natural, although not obvious a priori, considering that the present method is similar to the MK procedure. Therefore, the present finding should not be regarded as new evidence for the validity of the droplet picture. Rather, the present method should be used as a guide for other numerical methods with no approximation, such as Monte Carlo simulation, as one can easily draw a wrong conclusion from those methods because of severe limitation on the system size and relatively large corrections to scaling. The present result suggests that large systems (say, $L>32$ ) may be required in the three-dimensional case in order to get rid of the correction to scaling and obtain a reliable estimate of critical exponents. Another advantage of the present method, that it may enable us to compute dynamical quantities much more easily than using other methods, will be exploited in a future study.

A part of the numerical calculation in this work has been carried out using the facilities of the Supercomputer Center, Institute for Solid State Physics, University of Tokyo.

1) V. Cannella and J. A. Mydosh: Phys. Rev. B. 6 (1972) 4220.

2) S. F. Edwards and P. W. Anderson: J. Phys. F5 (1975) 965.

3) A. T. Ogielski: Phys. Rev. B 32 (1985) 7384.

4) R. N. Bhatt and A. P. Young: Phys. Rev. Lett. 54 (1985) 924.

5) N. Kawashima and A. P. Young: Phys. Rev. B 53 (1996) R484.

6) J. Houdayer: Eur. Phys. J. 22 (2001) 479.

7) J. Houdayer and A. K. Hartmann: Phys. Rev. B 70 (2004) 014418.

8) G. Parisi: Phys. Rev. Lett. 43 (1979) 1754.

9) D. S. Fisher and D. A. Huse: Phys. Rev. Lett. 56 (1986) 1601.

10) F. Barahona: J. Phys. A: Math. Gen. 15 (1982) 3241.

11) A.A. Migdal: Sov Phys. JETP 42 (1976) 743.

12) A. P. Young and R. B. Stinchcombe: J. Phys. C 9 (1976) 4419.

13) B. W. Southern and A. P. Young: J. Phys. C 10 ( 1977) 2179.

14) W. L. McMillan: Phys. Rev. B 29 (1984) 4026;

15) W. L. McMillan: Phys. Rev. B 30 (1984) 476.

16) A. J. Bray and M. A. Moore: in Heidelberg Colloquium on Spin Glasses, eds. J. L. van Hemmen and I. Morgenstern (Springer, Berlin, 1986) p. 121.

17) Four-point functions, which we need for the Binder parameter, can be reduced into products of two-point functions, analogous to Wick's theorem for the Gaussian integration.

18) S.-K. Ma, C. Dasgupta and C.-K. Hu: Phys. Rev. Lett. 43 (1979) 1434.

19) D. S. Fisher: Phys. Rev. Lett. 69, 534 (1992).

20) O. Motrunich, S.-C. Mau, D. A. Huse and D. S. Fisher: Phys. Rev. B 61 (2000) 1160.

21) Y.-C. Lin, N. Kawashima, F. Iglói and H. Rieger: Prog. Theor. Phys. Supplement 138 (2000) 479.

22) E. Marinari, G. Parisi and J. J. Ruiz-Lorenzo: Phys. Rev. B 58 (1998) 14852. 